\title{
Dependence of EMG-Reaction Times of the Rectus Femoris on Position Changes of the Hip Joint - Role of the Joint Capsule -
}

\author{
Norikazu Irie and Ryutchi Nakamura \\ Department of Rehabilitation Medicine, Institute of Balneology, \\ Tohoku University School of Medicine, Narugo, Miyagi 989-68
}

\begin{abstract}
IrIE, N. and Nakamura, R. Dependence of EMG-Reaction Times of the Rectus Femoris on Position Changes of the Hip Joint. - Role of the Joint CapsuleTohoku J. exp. Med., 1980, 131 (2), 207-208 — EMG-reaction times (EMG-RTs) of the rectus femoris in midposition (M) and internally rotated position (IR) of the right hip joint were examined in nine control subjects and nine patients who had lost most of the capsule of a hip joint after total hip joint replacement procedure. In both groups EMG-RTs of the rectus femoris were faster at IR than at $M$. The difference of EMG-RTs between $M$ and IR was essentially the same in the two groups. It is assumed that information from proprioceptors in the joint capsule may not play an important role in postural dependence of EMGRTs of the muscle.— reaction time; position changes of joint; joint capsule; proprioceptor
\end{abstract}

Postural changes and differences of the attitude influence the EMG reaction time (EMG-RT) of a limb muscle (Nakamura and Viel 1974). For example, EMG-RTs of the rectus femoris were usually faster at internally or externally rotated position of the hip joint than at midposition when normal subjects seated on a chair and responded to a peep sound with a rapid extention of the knee joint. The postural dependence of EMGRTs disappeared in patients with ventrolateral thalamotomy, cerebellar or frontal lesions. From these results it was assumed that information of position changes, arising from proprioceptors, was transmitted to these parts in the central nervous system and regulated the timing or the initiation of rapid movements (Nakamura 1976). Although proprioceptors locate in joints, tendons and muscles, it is not clear which proprioceptors participate in this mechanism. Recently there are patients who had lost of the capsule of the hip joint after total hip replacement procedure (Charnley 1979). Examining these subjects it is possible to know the contribution of capsular afferents to information of position changes (Grigg et al. 1973). In this study we analyzed EMG-RTs of the rectus femoris in patients with total hip replacement and in normal subjects and attempted to define the role of capsular sensory mechanism in the postural dependence of EMG-RT.

Experiments were performed on nine patients (six osteoarthritis, three rheumatoid arthritis), three male and six female, aged from 40 to 71 years and nine normal subjects, five male and four female, aged from 42 to 62 years. There was no significant difference between ages of both groups. The right hip was operated in all patients and postoperative periods were from 1 to 24 months (mean: 6.4 months). The unoperated side of the patients could not be used as the control due to the limited range of motion. The subject was seated on a chair with the trunk in the upright position and the feet detached from the floor. In this position the hip joint flexed at $90^{\circ}$ and rotated at $0^{\circ}$, and the knee joint flexed

Received for publication, October 24, 1979. 
at $90^{\circ}$ (midpostion $=M$ ). The subject was instructed to respond to a stimulus with a rapid extention of the right knee. The stimulus was a peep sound $(1000 \mathrm{~Hz}, 50 \mathrm{msec}$ duration) which was presented 2 to 4 second after a warning verbal signal. The EMG was recorded with surface electrodes attached on the right rectus femoris and displayed on a memoscope equipped with an averaging computer (Signal Processor 707TA Sanei). The record was analyzed by measuring with a millisecond scale the distance between the point on the EMG tracing where the stimulus began and the first electrical activity. EMG-RTs were measured in the following two positions of the hip joint held by an examiner: midposition as shown above and internal rotation about $30^{\circ}$ (IR). Before the experimental run, several trials were performed to acquaint the subject thoroughly with the procedure. The positions were changed every six trials and 18 trials performed in each position. The first trial after each position change was always discarded from data. The means and s.D. calculated from 15 trials in each position were used for statistical analysis.

In the normal subjects the means and S.D. of EMG-RTs were $119.2 \pm 11.4 \mathrm{msec}$ at $\mathrm{M}$ and $110.5 \pm 11.7 \mathrm{msee}$ at IR. The difference of EMG-RTs between $M$ and $\operatorname{IR}(\Delta(M-I R))$ was $8.7 \pm 9.4$ msec and statistically significant $(p<0.05, n=9)$. In the patients the means and s.D. were $140.4 \pm 27.5 \mathrm{msec}$ at $\mathrm{M}$ and $131.3 \pm 27.3 \mathrm{msec}$ at IR. $\Delta(\mathrm{M}-\mathrm{IR})$ was $9.1 \pm 10.1 \mathrm{msec}$ and statistically significant $(p<0.05, n=9)$. There was no significant difference between $\Delta(\mathrm{M}-\mathrm{IR})$ of the two groups $(p>0.9, n=18)$. There was no correlation between the postoperative periods and $\Delta(\mathrm{M}-\mathrm{IR})$ in the patients $(r=-0.38)$.

In the patients EMG-RTs of the rectus femoris were influenced by position changes of the hip joint similarly in normal subjects. Also the postoperative period did not correlate to $\Delta(M-I R)$. From these results it is assumed that information from proprioceptors in a joint capsule may not play an important role in the postural dependence of EMGRTs of a muscle. Lindström and Norrsell (1971), observing the locomotion of a cat in which the articular nerve of the knee was extensively transected in one hind limb, reported that the cat was moving rapidly and there was no difference of movements between the operated and the unoperated limb. Their report and our results suggest that information from the joint capsule is not necessary for the coordination of rapid movements.

\section{References}

1) Charnley, J. (1979) Low Friction Arthroplasty of the Hip. Springer-Verlag, BerlinHeidelberg-New York.

2) Grigg. P.. Fineman, G.A. \& Riley, L.H. (1973) Joint-position sense after total hip replacement. J. Bone Joint Surg., 55.A, 1016-1025.

3) Lindström, S. \& Norrsell, U. (1971) A note on knee joint denervation and postural reflexes in the cat. Acta physiol. scand., 82, 406-408.

4) Nakamura, R. (1976) Postural dependence of reaction time in normal subjects and patients with focal brain lesions. Appl. Neurophysiol., 39, 321-325.

5) Nakamura, R. \& Viel, E. (1974) The influence of position changes in the proximal joint on EMG-recorded reaction time on key muscles in the human extremity. In: Proc. 7 th Congr. of WCPT, pp. 119-123. 